\title{
Metodología indagatoria y rendimiento académico de estudiantes de la Universidad Continental, 2014
}

\author{
Research methodology and students' academic performance \\ in Universidad Continental, 2014
}

\author{
Roxana Pamela Espejo Ramos ${ }^{1}$ \\ Universidad Continental \\ roxanaespejo@gmail.com
}

\author{
Verónica Canales Guerra² \\ Universidad Continental \\ vcanales@continental.edu.pe
}

\section{RESUMEN}

El objetivo ha sido determinar el efecto que tiene la metodología indagatoria sobre el rendimiento académico (RA) de los estudiantes de la asignatura de biología de Universidad Continental. Para este fin se evaluó y comparó el RA de los estudiantes del grupo control y experimental antes de aplicar la metodología indagatoria; posteriormente se aplicaron sesiones de aprendizaje con la metodología indagatoria en el grupo experimental, finalmente se comparó el RA antes y después de la aplicación de la metodología indagatoria en el grupo experimental y grupo control. Los resultados fueron analizados mediante la prueba $F$ de Fisher $y$ t de Student. Entre los resultados se tiene que el RA pre test promedio del grupo control fue de 6,69 con desviación estándar (DE) de 1,948 y coeficiente de variación (CV) de 29,1 \%, mientras que en el grupo experimental fue de 6,66 con DE de 1,961 y CV de $29,7 \%$, por lo tanto el RA de ambos grupos fueron idénticos, homogéneos ( $C V>15 \%)$, con varianzas y $D E$ iguales. Respecto al RA en el grupo experimental en el post test, se obtuvo un promedio de 11,53 con DE de 2,874 y CV de 24,9\%, mediante la prueba t de Student al $95 \%$ de confianza estadística, se prueba que la media del RA del grupo experimental en el post test es mayor que la media respectiva en el pre test, pues el valor $P(\operatorname{Pr}(|\mathrm{T}|>|t|)=0)$ es menor que el nivel usual de significación de 0,05. En conclusión la metodología indagatoria tiene un efecto positivo sobre el rendimiento académico de los estudiantes de la asignatura de Biología de la Universidad Continental.

Palabras clave: Metodología indagatoria, rendimiento académico

\section{ABSTRACT}

The objective was to determine the effect that research methodology has on academic students' performance (SP) majoring in biology at the Universidad Continental. For this purpose, before applying the research methodology, a control and experimental group of students SP were evaluated and compared; afterwards learning sessions about research methodology were applied in the experimental group. Finally, SP was compared before and after the research methodology application in both groups. The results were analyzed using $F$ Fisher and t Student test. Normality was analyzed according to normality Shapiro test - Wilk and the homogeneity, according to F Fisher test. Results showed that the average pre SP test was 6, 69 for the control group with a 1,948 standard deviation (SD) and $29,1 \%$ coefficient of variation (CV), whereas the experimental group was 6,66 with a 1,961 standard deviation (SD) and $29,7 \%$ CV. Therefore, the SP in both groups were identical, homogeneous (CV> 15 $\%)$, with equal variances and (SD). Respect to the SP in the experimental group in the post test, an average of 11,53 with a 2,874 (SD) was obtained and 24,9 $\%$ CV by Student $t$ test at $95 \%$ confidence, which proves that the average SP of the experimental group in the post-test is greater than the average in the respective pretest, since the $P$ value $(\operatorname{Pr}(|T|>|t|)=$ 0 ) is less than the usual significance level of 0,05 . In conclusion, the research methodology has a positive effect on the academic students' performance in Biology class, at the Universidad Continental.

Keywords: Research methodology, academic performance.

Historial del artículo:

Recibido: 16 de agosto de 2015. Aprobado: 12 de noviembre de 2015. Disponible en línea: 30 de diciembre de 2015

1 Magister en Educación, mención Enseñanza Estratégica; docente de la Universidad Nacional del Centro del Perú.

2 Biologa; docente de la Universidad Continental; investigadora de la Universidad Continental. 


\section{INTRODUCCIÓN}

La realidad laboral actual exige ciudadanos que se encuentren en la capacidad de tener una participación más significativa en su entorno social; así; las universidades buscan dar respuesta a esta necesidad intentado formar profesionales competentes con miras a incorporarlos en la estructura social y económica del país; sin embargo; los resultados que se obtienen no siempre resultan tener el impacto deseado. Prueba de ello es el estudiante universitario promedio, que recurre a estrategias de aprendizaje que respondan a las necesidades del momento, a obtener una buena calificación o incluso llegar a una nota mínima aprobatoria, hablamos entonces de estrategias ineficientes, que no promueven un aprendizaje significativo que se centran en el memorismo, reduciendo el proceso de enseñanza-aprendizaje a la obtención de números y no competencias.

Por otro lado, tenemos al docente, que puede o no haber tenido una formación pedagógica, pero que finalmente ejerce la docencia y se ve en la necesidad de responder a los requisitos de un sistema académico que le exige diseñar sesiones de aprendizaje, unidades de aprendizaje, elaborar instrumentos de evaluación, es decir, invertir gran parte del tiempo en labores administrativas y no solo pedagógicas.

En medio de todo este contexto el docente se pregunta sobre las estrategias o métodos que debe emplear para enseñar, volvemos a recordar el hecho de que puede o no haber tenido formación pedagógica, lo cual suele ser una gran debilidad, sobre todo porque son las estrategias de enseñanza las que nos permiten tener claro el horizonte al cual queremos llegar.

Siendo aún más específicos y centrándonos en la enseñanza de las ciencias, como biología, química, anatomía, botánica, etc., se ve la necesidad de relacionar la teoría con la práctica, de allí el hecho de que sea muy importante contar con laboratorio equipados. Sin embargo, regresamos a la importancia de la selección de un adecuado método o estrategia de enseñanza que permita efectivizar el aprendizaje.

En vista de esta problemática, hace algunos años se viene incorporando el método indagatorio en la enseñanza de las ciencias, a fin de promover aprendizajes significativos en los estudiantes de educación básica regular y universitaria, promoviendo de este modo el desarrollo de habilidades científicas que le permitan por ejemplo, plantear hipótesis, experimentar, registrar observaciones, analizar los resultados, plantear conclusiones y aplicar el nuevo conocimiento en la solución de problemas del contexto. De modo que, una sesión de aprendizaje pone al estudiante en rol de investigador y constructor de su aprendizaje.
Es evidente que la aplicación de la metodología indagatoria promueve el desarrollo del pensamiento científico y permite optimizar el carácter experimental de las ciencias como un medio para aprender a aprender. Por esto es conveniente enseñar ciencia haciendo ciencia; es decir; orientar a los estudiantes a pensar, a aprender, a potenciar sus mentes curiosas e imaginativas, de modo de que cada aprendizaje sea significativo y trascienda en el tiempo.

(1) La idea central de la metodología indagatoria es propiciar una estrategia de enseñanza y aprendizaje que parta de la observación de la realidad, interacción con problemas concretos, propiciándose preguntas referentes a esa realidad que promuevan la búsqueda de información y la experimentación, por ende la construcción activa de su aprendizaje. La aplicación de esta metodología requiere de un proceso sistemático, por lo que a lo largo de su aplicación en diferentes países, se ha requerido de componentes y etapas específicas durante su implementación, es así que se consideran cuatro etapas importantes:

La etapa de focalización, es la primera etapa, por ende la crucial para el desarrollo de la metodología, en ella se debe propiciar el interés y la motivación en el estudiante sobre una situación problema. Está basada en la contextualización de una situación, esto se puede dar mediante la observación, el relato de un evento de la comunidad o la presentación de una situación desconocida, seguida de una pregunta bien diseñada que promueva el interés de los estudiantes y la necesidad de resolverla. Su desarrollo debe ser individual, a modo de extraer las concepciones y conocimientos previos que posee el estudiante sobre el tema central del problema, y hacer los ajustes pertinentes en su planificación para lograr una construcción efectiva del conocimiento.

Así, en una clase de genética se podría presentar el siguiente caso:

"El factor Rh es una proteína integral de la membrana de los glóbulos rojos (GR), de modo que son Rh (+) quienes presentan dicha proteína y $R h(-)$ quienes no la presentan. Un $85 \%$ de la población son Rh (+). Dentro de las recomendaciones médicas antes o durante el embarazo, las mujeres deben realizar un test para conocer su grupo sanguíneo y el factor Rh para evitar abortos espontáneos en las mujeres que son Rh (-) sobre todo a partir del segundo embarazo."

\section{Preguntas de focalización:}

¿Por qué es importante que las mujeres conozcan su factor Rh?

¿Por qué será más importante a partir del segundo embarazo? 
¿Será necesario también conocer el factor Rh del padre, por qué?

¿Qué recomendaciones daría a una mujer con factor Rh (-), por qué?

Recordemos que este tema está orientado a estudiantes de ciencias de la salud, de la asignatura de biología, de este modo, los ponemos a resolver una situación problemática que será parte de su contexto laboral, por lo tanto los ponemos en el rol de profesionales de la salud y no solo de receptores de conocimientos. En esta etapa los estudiantes plantean sus hipótesis o posibles respuestas, valiéndose de sus conocimientos previos, su sistema de creencias y su visión de la ciencia.

La etapa de exploración, es la que va a propiciar el aprendizaje, en ella los estudiantes desarrollan su investigación, se fundamentan en sus ideas y buscan estrategias para desarrollar experiencias que los lleven a conseguir resultados. Es importante que los estudiantes elaboren sus procedimientos y el docente sirva sólo de guía, permitiendo la argumentación, razonamiento y confrontación de sus puntos de vista.

En el mismo caso, será necesario brindar al estudiante información sólida sobre el factor Rh, el modo de herencia de este factor (recesivo o dominante), investigaciones actuales y estadísticas de su localidad, que le permitan comprender la reacción entre el factor Rh (-) materno y Rh (+) fetal. Esto puede resultar intrascendente en el primer embarazo pues la madre no esta sensibilizada frente a la presencia de estas proteínas, pero puede ser fatal para el feto en un segundo embarazo, pues el organismo de la madre actúa como una máquina asesina (por la presencia de anticuerpos contra el factor Rh (+) fetal), provocando hemolisis en el feto, fenómeno conocido como eritroblastosis fetal. Esto lleva al aborto espontaneo o al nacimiento de bebés prematuros con extrema ictericia que puede llegar a afectar su sistema nerviosos central.

La etapa de comparación o reflexión, se requiere la participación activa del estudiante. El deberá confrontar la realidad de los resultados observados con sus predicciones, formulando sus propias conclusiones. El docente por su parte, debe estar atento para introducir términos y conceptos que considere adecuados, mediar para que el estudiante reflexione y analice detalladamente sus conclusiones, utilizando preguntas que las cuestione. Las conclusiones deben presentarse de forma oral y escrita, donde el estudiante incluya los conceptos y términos que crea necesarios.

En esta etapa se vuelve a plantear las preguntas de la focalización, sin embargo, con la nueva información adquirida, el estudiante contrastará sus hipótesis, dará respuestas sobre una base científica, se convertirá en un profesional de la salud, dando orientación clínica a su paciente.

La etapa de aplicación, es la confirmación del aprendizaje, en ella el estudiante debe ser capaz de extrapolar el aprendizaje a eventos cotidianos, generando investigaciones o extensiones del trabajo experimental.

En la aplicación, se puede dar respuesta a las interrogantes surgidas durante la clase, por ejemplo, puede ser que no se cuente con datos estadísticos sobre los casos de eritroblastosis fetal en la ciudad, se puede promover entonces el desarrollo de una investigación descriptiva, que surge desde la necesidad de comprender este fenómeno en nuestro contexto. Los estudiantes pueden solicitar asesoría de pediatras y ginecólogos. Así se pasa de una educación tradicional y memorista a una que adquiere mayor significado y trascendencia en la vida del estudiante, desarrollando en él competencias investigativas.

La metodología indagatoria pretende pasar del paradigma del aprendizaje de contenidos, al desarrollo de competencias científicas (2) que alude a la capacidad y la voluntad de utilizar el conjunto de conocimientos y la investigación científica para explicar la naturaleza y actuar en contextos de la vida real. Entendemos por competencia en la cultura científica a aquel conocimiento científico y el uso que se hace de ese conocimiento para identificar cuestiones, adquirir nuevos conocimientos, explicar los sistemas y fenómenos naturales más relevantes, la forma en que el entorno condiciona las actividades humanas, las consecuencias de esas actividades en el medio ambiente, las aplicaciones y desarrollos tecnológicos de la ciencia, actuar consciente y eficazmente en el cuidado de la salud personal y extraer conclusiones basadas en pruebas sobre temas relacionados con las ciencias y su aplicación práctica en la vida cotidiana. Por lo tanto, esta competencia se centra, tanto en el conocimiento científico y el uso del mismo que hace posible actuar e interactuar de manera significativa en situaciones en las cuales se requiere producir, apropiar o aplicar comprensiva y responsablemente los conocimientos científicos, como en resolver problemas de naturaleza científica y tecnológica, así como analizar críticamente la forma en que ciencia y tecnología influyen en el modo de vida de la sociedad actual.

Para este fin emplea la Indagación científica que (3) se define como un proceso en el cual "se plantean preguntas acerca del mundo natural, se generan hipótesis, se diseña una investigación, se recolectan y analizan datos con el objeto de encontrar una solución al problema". La indagación, es un proceso que explora el mundo natural o material, lo que lleva 
a formular hipótesis, experimentar, conjeturar y hacer descubrimientos. El proceso indagatorio es manejado por la propia curiosidad, el interés, las preguntas y la pasión por explicar una observación o resolver un problema. La enseñanza por indagación resalta la importancia fundamental de que los estudiantes puedan implicarse personalmente en el camino de "hacer ciencia". En otras palabras, aprender ciencia requiere participar activamente en la recolección de información a través de los sentidos: ver, escuchar, tocar, gustar y oler. La indagación incentiva a los estudiantes a preguntar, llevar a cabo investigaciones y hacer sus propios descubrimientos, hace uso de múltiples formas de saber y adquirir nuevas perspectivas al explorar temas, contenidos y preguntas. La enseñanza de las ciencias basada en la indagación privilegia la experiencia y los conocimientos previos, su práctica es transformadora tanto para el docente como para los estudiantes, toma en cuenta algunos nuevos aportes en la enseñanza que insisten en la necesidad de que los estudiantes desempeñen un papel más activo durante el desarrollo de las sesiones de aprendizaje.

Otro concepto que de importancia para la aplicación de la metodología indagatoria es la Alfabetización científica (4), que se entiende como "la capacidad de usar el conocimiento científico para identificar preguntas y para sacar conclusiones basadas en las pruebas, con el fin de entender y ayudar a tomar decisiones sobre el mundo natural y los cambios realizados en él a través de la actividad humana"

Descrita la metodología indagatoria, es oportuno comprender las teorías que la sustentan. Encontramos así, (5) el modelo constructivista del aprendizaje, un movimiento contemporáneo que sintetiza tanto el desarrollo de las modernas teorías del aprendizaje como el de la psicología cognitiva; que se opone a concebir el aprendizaje como receptivo y pasivo considerándolo más bien, como una actividad organizadora, compleja del alumno que construye y reconstruye sus nuevos conocimientos propuestos, a partir de revisiones, selecciones, transformaciones y reestructuraciones de sus antiguos conocimientos pertinentes, en cooperación con su maestro y sus maestros y compañeros; es decir el verdadero aprendizaje humano es una construcción de cada quien, el cual logra modificar su estructura mental. Por otra parte la (6) la teoría del aprendizaje por descubrimiento planteada por el psicólogo Bruner considera que "el pensamiento es mejorable a través del aprendizaje por descubrimiento y de la acción y ello pasa por las fases, enactiva, icónica y simbólica". Los estudiantes logran sus conocimientos empleando diversos recursos de su entorno, exponiendo, interpretando conceptos, símbolos, analizando los procesos físicos y biológicos de las situaciones planteadas en el aula o fuera de ella. Así mismo la (7) La teoría del aprendizaje significativo es un proceso por medio del que se relaciona la nueva información con algún aspecto ya existente en la estructura cognitiva del individuo y que sea relevante para el material que se necesita aprender. Desde esta perspectiva, "el aprendizaje debe ser necesariamente significativo para el estudiante, si queremos que represente algo más que palabras o frases que repite de memoria y que definitivamente se olvidarán."

Así, el constructivismo, el aprendizaje por descubrimiento y la teoría del aprendizaje significativo, confluyen para dar un sustento teórico sólido a la metodología indagatoria.

De este modo, se evidencian experiencias y antecedentes respecto a la aplicación de la metodología indagatoria y si esta tiene efectos o no sobre el rendimiento académico de los estudiantes.

(8) En el modelo tradicional de enseñanza, el docente es quien provee los conocimientos elaborados y el alumno, en forma pasiva los consume, sin asegurar la transmisión del saber. Los modelos de enseñanza mediante la investigación postulan la importancia de situar al alumno en un contexto similar al que se encuentra un científico, pero bajo la dirección de un docente. (9) En el 2008 tras un estudio sobre "La metodología indagatoria como herramienta para explicitar preconceptos sobre orientaciones espaciales en estudiantes de universitarios en ciencias" se pudo evidenciar que si bien los estudiantes universitarios se encuentran en niveles de conocimiento "superiores" a los de estudiantes secundarios, sus representaciones espaciales siguen siendo como lo son aquellas de estudiantes de 14 años de edad. Esto debido probablemente al hecho de que la enseñanza de la física no ha sido abordada de modo contextualizado, ya que no se crean las condiciones para que los estudiantes desarrollen sus habilidades especiales y científicas a partir de los saberes previos. En este mismo se considera que la metodología de enseñanza llamada indagación, se ha instalado en las aulas de educación básica, media y universitaria, por su característica de "construcción del aprendizaje" desde la activación del pensamiento, y en este trabajo se pretende enfatizar también, a esta metodología de enseñanza, la característica de facilitadora de investigaciones educativas.

(10) George Charpack, Premio Nobel de Física, 1992, es el creador del Programa "La Main a la Pate", (Las manos en la masa) programa propuesto para la puesta en práctica de la metodología indagatoria, la cual promueve una enseñanza que no sea repetitiva ni mucho menos memorística, sino más bien, una educación basada en la observación, la experimentación, la argumentación y el razonamiento. De este modo este programa busca acercar la ciencia a las aulas, logrando fortalecer el aprendizaje de los 
estudiantes en cuando a las ciencias se refiere. (11) Investigaciones realizadas en el nivel universitario, han aportado resultados valiosos respecto a la respuesta de los estudiantes luego de implementar intervenciones didácticas en busca de reorientar la formación científica de los estudiantes, lo que eventualmente permitió dotar al docente de herramientas que le permitieron comprender los principios de la alfabetización científica. Estos resultados fueron obtenidos luego de 11 años de emplear estrategias dirigidas al logro del aprendizaje significativo, a partir del desarrollo de habilidades de razonamiento crítico y creativo, componentes del pensamiento científico y de una educación para la vida.

Por estas razones, el presente investigación quiso incorporar la metodología indagatoria en la enseñanza de la biología, dirigida a estudiantes universitarios. Con esto se espera desarrollar habilidades científicas en los mismos, despertando en ellos la curiosidad nata de todo investigador, que encuentra en las ciencias un medio para conocer la realidad que lo rodea.

Así, la investigación parte de la siguiente pregunta: ¿Qué efecto tiene la metodología indagatoria sobre el rendimiento académico de los estudiantes de la asignatura de biología de Universidad Continental? . La hipótesis planteada fue que la metodología indagatoria tiene un efecto positivo sobre el rendimiento académico de los estudiantes de la asignatura de biología de Universidad Continental.

El objetivo general fue determinar el efecto que tiene la metodología indagatoria sobre el rendimiento académico de los estudiantes de la asignatura de biología de Universidad Continental. Los objetivos específicos fueron determinar la homogeneidad del rendimiento académico de los grupos en el pre test, para que; luego de aplicar la metodología indagatoria; se evalué la diferencia del rendimiento académico de los dos grupos en el post test y la diferencia del rendimiento académico del grupo experimental entre el pre y post test. Finalmente determinar la eficacia del tratamiento experimental, para inferir su efecto sobre el rendimiento académico de los estudiantes.

\section{MATERIAL Y MÉTODOS}

Se trató de una investigación cuantitativa, de tipo aplicada, nivel experimental y diseño cuasi experimental. La población estuvo conformada por 200 estudiantes de la Facultad de Ciencias de la Salud, matriculados en la asignatura de biología. La muestra estuvo conformada por 29 estudiantes para el grupo control y 32 estudiantes para el grupo experimental. Se analizó la normalidad, según la prueba de normalidad de Shapiro - Wilk y, las varianzas del rendimiento académico de los grupos control y experimental para homogeneidad, según la prueba $F$ de Fisher, a fin de determinar el grupo control y experimental. Se seleccionó el grupo experimental al cual se aplicaron las sesiones de aprendizaje basadas en la metodología indagatoria, considerando los procesos de focalización, exploración, reflexión y aplicación. Las sesiones fueron previamente socializadas con especialistas del Ministerio de Educación del programa de especialización en Ciencia Tecnología y Ambiente. Para el rendimiento académico se aplicaron pruebas pre test y post test previamente validadas por juicio de expertos, los resultados fueron analizados mediante la prueba $\mathrm{F}$ de Fisher para la razón de varianzas y la prueba $t$ de Student para la homogeneidad de medias de muestras independientes con distribuciones normales, reportadas con el programa estadístico Stata V.13.

\section{RESULTADOS}

\section{Análisis del rendimiento académico en el pre test}

En la tabla 1 se muestra que, en el pre test, la mayoría de los estudiantes del grupo control (76\%) y grupo experimental (69\%) tienen rendimiento académico de 6 a 10, el $21 \%$ de los primeros y el $28 \%$ de los segundos entre 0 y 5 y, el $3 \%$ de ambos grupos entre 11 y 15 . Estos porcentajes indican que, en el pre test, no existen diferencias significativas en el rendimiento académico de los estudiantes de ambos grupos de estudio.

En la tabla 1A se observa que, en el pre test, el rendimiento académico de ambos grupos fluctúa entre 3 y 11 y, con puntajes frecuentes de 6 . El $50 \%$ de los estudiantes del grupo control tiene como máximo 7 y el $50 \%$ de los estudiantes del grupo experimental 6,5.

De estos estadísticos se deriva que, en el pre test, los rendimientos académicos de ambos grupos son idénticos, heterogéneos (CV > $15 \%$ ) (Chue et al., 2009: 59), con medias, varianzas y desviaciones estándares iguales.

Estas aseveraciones son confirmadas con la prueba $F$ de Fisher para la razón de varianzas y la prueba t de Student para la homogeneidad de medias de muestras independientes con distribuciones normales. Con la primera prueba se establece que, al $95 \%$ de confianza estadística, las varianzas del rendimiento académico de los grupos control y experimental son iguales, pues el valor $\mathrm{P}(2 * \operatorname{Pr}(\mathrm{F}<\mathrm{f})=0,9398)$ es mayor que el nivel usual de significación de 0,05 . Con la segunda prueba se afirma que las medias del rendimiento académico de los grupos control y experimental son iguales, pues el valor $P(\operatorname{Pr}(|\mathrm{T}|>|t|)=0,9473)$ es mayor que el nivel usual de significación de 0,05. 
Las distribuciones del rendimiento académico de los estudiantes de ambos grupos son simétricas (CA entre $-0,5$ y 0,5$)$ y de la misma altura que la distribución normal (curvas mesocúrticas o curvas MK, CC entre $-0,5$ y 0,5$)$. Estos resultados son confirmados con la prueba de normalidad de Shapiro-Wilk, al $95 \%$ de confianza estadística, donde los valores $\mathrm{P}$ son mayores que el nivel usual de significación de 0,05.

\section{Análisis del rendimiento académico en el post test}

En la tabla 2 se aprecia que, en el post test, la mayoría de los estudiantes del grupo control (79\%) y el $44 \%$

Tabla $N^{\circ}$ 1: Rendimiento académico de los estudiantes en el pre test por grupo de estudio.

\begin{tabular}{lcccc}
\hline \multirow{2}{*}{$\begin{array}{c}\text { Rendimiento } \\
\text { académico }\end{array}$} & \multicolumn{2}{c}{$\begin{array}{c}\text { Grupo control } \\
(\mathbf{n}=29)\end{array}$} & $\begin{array}{c}\text { Grupo experimental } \\
(\mathbf{n}=32)\end{array}$ \\
\cline { 2 - 5 } & Estudiantes & $\%$ & Estudiantes & $\%$ \\
\hline 0 a 5 & 6 & 21 & 9 & 28 \\
6 a 10 & 22 & 76 & 22 & 69 \\
11 a 15 & 1 & 3 & 1 & 3 \\
\hline
\end{tabular}

Tabla № 1A: Estadísticos del rendimiento académico de los estudiantes en el pre test por grupo de estudio.

\begin{tabular}{lrr}
\hline Estadístico & $\begin{array}{r}\text { Grupo } \\
\text { control }\end{array}$ & $\begin{array}{r}\text { Grupo } \\
\text { control }\end{array}$ \\
\hline Estudiantes & 29,000 & 32,000 \\
Mínimo & 3,000 & 3,000 \\
Máximo & 11,000 & 11,000 \\
Moda & 6,000 & 6,000 \\
Mediana & 7,000 & 6,500 \\
Media & 6,690 & 6,660 \\
Desviación estándar (DE) & 1,948 & 1,977 \\
Coeficiente de variación (CV) & $29,10 \%$ & $29,70 \%$ \\
Coeficiente de asimetría (CA) & $-0,058$ & 0,273 \\
Coeficiente de curtosis (CC) & $-0,036$ & $-0,449$ \\
\hline
\end{tabular}

del grupo experimental tienen rendimiento académico de 6 a 10 , el $14 \%$ de los primeros y el $47 \%$ de los segundos entre 11 y 15 , el $7 \%$ de los primeros entre 0 y 5 y, el $9 \%$ de los segundos entre 16 y 20 . Estos porcentajes indican que, en el post test, existen diferencias significativas en el rendimiento académico de los estudiantes de los grupos de estudio, favorables al grupo experimental, como efecto de la metodología indagatoria empleada en este grupo.

En la tabla $2 \mathrm{~A}$ se observa que, en el post test, el rendimiento académico del grupo control fluctúa entre 3 y 13 y, con puntajes frecuentes de 8 . El $50 \%$ de los estudiantes de este grupo tiene como máximo 8 . Por su parte, el rendimiento académico del grupo control fluctúa entre 7 y 18 y, con puntajes frecuentes de 10 . El $50 \%$ de los estudiantes de este grupo tiene como máximo 11 .

El rendimiento académico promedio del grupo controles de 8,07 con desviación estándar de 2,219 y coeficiente de variación de $27,5 \%$, mientras que el rendimiento académico promedio del grupo experimental es de 11,53 con desviación estándar de 2,874 y coeficiente de variación de $24,9 \%$. De estos estadísticos se deriva que, en el post test, los rendimientos académicos de ambos grupos son distintos, heterogéneos (Chue et al., 2009: 59), con medias distintas pero varianzas y desviaciones estándares iguales.

Tabla № 2: Rendimiento académico de los estudiantes en el post test por grupo de estudio.

\begin{tabular}{lcrcr}
\hline $\begin{array}{c}\text { Rendimiento } \\
\text { académico }\end{array}$ & \multicolumn{2}{c}{$\begin{array}{c}\text { Grupo control } \\
(\mathbf{n}=29)\end{array}$} & $\begin{array}{c}\text { Grupo experimental } \\
(\mathbf{n}=32)\end{array}$ \\
\cline { 2 - 5 } & Estudiantes & $\%$ & Estudiantes & $\%$ \\
\hline 0 a 5 & 2 & 7 & & \\
6 a 10 & 23 & 79 & 14 & 44 \\
11 a 15 & 4 & 14 & 15 & 47 \\
16 a 20 & & & 3 & 9 \\
\hline
\end{tabular}

Tabla №2A: Estadísticos del rendimiento académico de los estudiantes en el post test por grupo de estudio.

\begin{tabular}{lrr}
\hline Estadístico & $\begin{array}{r}\text { Grupo } \\
\text { control }\end{array}$ & $\begin{array}{c}\text { Grupo } \\
\text { experimental }\end{array}$ \\
\hline Estudiantes & 29,000 & 32,000 \\
Mínimo & 3,000 & 7,000 \\
Máximo & 13,000 & 18,000 \\
Moda & 8,000 & 10,000 \\
Mediana & 8,000 & 11,000 \\
Media & 8,070 & 11,530 \\
Desviación estándar (DE) & 2,219 & 2,874 \\
Coeficiente de variación (CV) & $27,50 \%$ & $24,90 \%$ \\
Coeficiente de asimetría (CA) & 0,118 & 0,470 \\
Coeficiente de curtosis (CC) & 0,646 & $-0,045$ \\
\hline
\end{tabular}

Estas aseveraciones son confirmadas con la prueba F de Fisher para la razón de varianzas y la prueba † de Student para la homogeneidad de medias de muestras independientes con distribuciones normales. Con la primera prueba se establece que, al $95 \%$ de confianza estadística, las varianzas del rendimiento académico de los grupos control y experimental son iguales, pues el valor $\mathrm{P}(2 * \operatorname{Pr}(\mathrm{F}<\mathrm{f})=0,1705)$ es mayor que el nivel usual de significación de 0,05 (figura 2B). Con la segunda prueba se afirma que la media del rendimiento académico del grupo experimental es mayor que la media respectiva del grupo y experimental, pues el valor $\mathrm{P}(\operatorname{Pr}(\mathrm{T}>\mathrm{t})=0)$ es menor que el nivel usual de significación de 0,05. 
Las distribuciones del rendimiento académico de los estudiantes de ambos grupos son simétricas, la primera más alta que la distribución normal (curva leptocúrtica o curva LK, CC > 0,5) y la segunda de la misma altura que la distribución normal (curva MK). Estos resultados son confirmados con la prueba de normalidad de Shapiro-Wilk, al $95 \%$ de confianza estadística, donde los valores $\mathrm{P}$ son mayores que el nivel usual de significación de 0,05.

\section{Contrastación estadística de hipótesis}

Hipótesis:

"La metodología indagatoria tiene efectos positivos sobre el rendimiento académico de los estudiantes de la asignatura de Biología de la Universidad Continental".

Dado que el diseño de investigación es cuasi experimental pre y post test con grupo control, la contrastación estadística de la hipótesis de investigación se realizó en cinco etapas:

a. Homogeneidad del rendimiento académico de los grupos en el pre test.

b. Diferencia del rendimiento académico de los grupos en el post test, favorable al grupo experimental.

c. Comparación del rendimiento académico del grupo control entre el pre y post test.

d. Diferencia del rendimiento académico del grupo experimental entre el pre y post test, favorable al pos test.

e. Si el rendimiento académico en el post test del grupo control es mayor al rendimiento académico en el pre test, evaluación de la eficacia del tratamiento experimental.

\section{DISCUSIÓN}

Respecto al RA comparativo en los grupos control y experimental luego de aplicada la metodología indagatoria, aceptan la hipótesis alternativa $\mathrm{Hl}$, al $95 \%$ de confianza estadística, que concluye que existen diferencias significativas en los rendimientos académicos promedios en el post test de los estudiantes de los grupos control y experimental, favorables al grupo experimental, por lo que la metodología indagatoria tiene un efecto positivo sobre el rendimiento académico de los estudiantes de la asignatura de biología de Universidad Continental

Los datos obtenidos son concordantes con otros estudios realizados (9) que abordaron "la metodología indagatoria como herramienta para explicitar preconceptos sobre orientaciones espaciales en estudiantes de universitarios en ciencias" evidenciando que los estudiantes desarrollan sus habilidades especiales y científicas a partir de los saberes previos (...) que la metodología indagatoria se ha instalado en las aulas por su característica de "construcción del aprendizaje" desde la activación del pensamiento como facilitadora de investigaciones educativas.

De igual manera (10) el programa "las manos en la masa" propuesto para la puesta en práctica de la metodología indagatoria, promueve una educación basada en la observación, la experimentación, la argumentación y el razonamiento, que obtuvo niveles de efectividad concordantes con los hallados en esta investigación (11). Asímismo, investigaciones realizadas en el nivel universitario, han aportado resultados valiosos respecto a la respuesta de los estudiantes luego de implementar intervenciones didácticas en busca de reorientar la formación científica de los estudiantes, lo que eventualmente permitió dotar al docente de herramientas que le permitieron comprender los principios de la alfabetización científica. Estos resultados fueron obtenidos luego de 11 años de emplear estrategias dirigidas al logro del aprendizaje significativo, a partir del desarrollo de habilidades de razonamiento crítico y creativo, componentes del pensamiento científico y de una educación para la vida.

Por lo tanto, se puede determinar que la metodología indagatoria tiene un efecto positivo sobre el rendimiento académico de los estudiantes, que es resultado de la práctica de habilidades científicas en la resolución de casos contextualizados orientados a su rol profesional.

Finalmente, es recomendable la aplicación de la metodología indagatoria desde los primeros semestres de formación universitaria a fin de poder promover en los estudiantes el desarrollo de habilidades y competencias científicas que permitan una sólida formación universitaria.

\section{REFERENCIAS BIBLIOGRÁFICAS}

1. Uzcátegui Y., Betancourt C. La metodología indagatoria en la enseñanza de las ciencias: una revisión de su creciente implementación a nivel de Educación Básica y Media. Revista de Investigación № 78 Vol. 37. Enero-Abril 2013

2. Departamento de Investigación, Universidades e Investigación del gobierno Vasco. Competencia en cultura científica, tecnológica y de la salud. Ubicado en http://ediagnostikoak.net/ediag/ cas/materiales-informativos/ED 11 _marko_ 
teorikoak/3_Competencia_cientifica.pdf

3. Programa de especialización en ciencia, tecnología y ambiente dirigido a docentes de instituciones educativas públicas del nivel de educación secundaria de educación básica regular (2012 - 2014)

4. Cajas F. Alfabetización científica y tecnológica: la transposición didáctica del conocimiento tecnológico. (American Association for the Advancement of Science, AAAS). Washington DC. ENSEÑANZA DE LAS CIENCIAS, 2001, 19 (2), 243-254 Ubicable en: http://ddd.uab.es/pub/ edlc/02124521v19n2p243.pdf

5. Quintana, C. y Cámac Z. Corrientes pedagógicas contemporáneas. Lima: Ed. San Marcos. 2007.

6. Morán JJ. Habilidades docentes en el desarrollo pedagógico. DLN² 2012-16361 Huancayo Ed Sol Graf. 2013

7. Ausubel DP. Psicología educativa. Un punto de vista cognoscitivo. México D. F.: Trillas. 1976

8. Loreto AM. MuñozFundamentos y Perspectivas de la Metodología indagatoria en la Enseñanzade las Ciencias. Material en línea. 2010 (citado el 20 de junio de 2014) Ubicable en: http://es.scribd.com/ doc/139424230/Metodologia-indagatoria-en-laensenanza-de-las-ciencias

9. Muñoz LA. La metodología indagatoria como herramienta para explicitar preconceptos sobre orientaciones espaciales en estudiantes de universitarios en ciencias. Rev.Col.Fís., Vol. 43, No. 3 de 2011.

10. Aviles G. La metodología indagatoria: una mirada hacia el aprendizaje significativo desde "Charpack y Vygotsky". InterSedes. Vol. XII. (23-2011) 133144. ISSN: 2215-2458

11. Villaruel F. Formación científica y desarrollo de competencias para la investigación experimental en las ciencias agrícolas. Umbral Editorial, S.A. ISBN: 978-607-619-065-4. Ubicable en: http://cenid. org.mx/libros/colecciones/practicas_educativas_ desde_la_perspectiva/libro.pdf\#page $=55$ 